\title{
Tactile apparent movement: The effects of shape and type of motion
}

\author{
JACOB H. KIRMAN \\ Queens College of the City University of New York
}

\begin{abstract}
Judgments of tactile apparent movement were obtained from six subjects for a variety of tactile spatiotemporal stimulus patterns (moving points and lines, rotating lines, and expanding squares, boundaries, and holes). Each of these patterns was presented by means of three different numbers of steps used to trace the pattern over time. Stimulus duration and interstimulus onset interval (ISOI) were varied to determine the temporal conditions required for good tactile apparent movement. In general, pattern shape and type of movement (linear, rotating, expanding) had little effect on the temporal conditions for good apparent movement or on the interactions among the other variables. Increasing the number of steps in a pattern decreased the ISOI required for good apparent movement, but had no effect on the range of ISOIs over which good movement was obtained. Increases in stimulus duration increased the ISOI required for good tactile apparent movement, in agreement with earlier studies, and increased as well the range of ISOIs over which good movement was obtained.
\end{abstract}

This experiment was stimulated by both theoretical and practical concerns. The theoretical interest was to examine the effects of shape and type of movement on the temporal conditions for the phenomenon of apparent movement in the tactual modality, as has been done in vision (Anstis, 1970, 1978; Kolers, 1972). The practical concern was to provide designers of tactile displays with an understanding of the proper spatiotemporal conditions for the emergence of good apparent movement under conditions involving tactile patterns of varying complexity, such as might result from a tactile display of speech. In particular, as I have discussed in an earlier paper (Kirman, 1973), it may be of considerable importance for a tactile display of speech to maintain a sense of continuity for some portions of the tactile stimulus stream (e.g., over syllabic units) while providing segmenting intervals that result in clear perceptual segregation at other times. It is obviously relevant to know whether the required temporal conditions for the illusion of continuous movement vary significantly with the geometry of the shapes presented or the type of motion described.

Previous experiments on tactile apparent movement have focused on only two point-stimulators or several such point-stimulators in a linear arrangement (Kirman, 1974a, 1974b, 1975; Sherrick \& Rogers, 1966). Whether more complex pattern shapes or directions of movement would be integratable by apparent movement at the same or similar temporal intervals was not clear. The present experiment, therefore, while including linear movement similar to that previously studied, also utilized several other shapes and types

The author's mailing address is: Department of Psychology, Queens College of CUNY, Flushing, New York 11367. of motion. An additional aspect of tactile display presentation investigated here was the number of successive steps involved in presenting any given spatiotemporal pattern. For example, in presenting the trajectory of a tactile stimulus over some distance from locus $A$ to locus $B$, one may present any number of intermediate points along the path from A to B. If one could produce a satisfactory perception of the desired movement with a minimum number of such intervening sequential steps, that would obviously result in a more efficient tactile display from the point of view of the time required to produce the desired effect. The present study was not, however, concerned with determining the minimum number of such intermediate steps required to produce a given motion effect. The focus here was, rather, on the effects of variation in the number of such intervening steps on the temporal conditions required for good apparent movement. In particular, this study investigated the effects of shape, type of movement, number of steps, and stimulus duration on the interstimulus onset intervals required to produce good apparent movement.

\section{METHOD}

\section{Apparatus}

All stimuli were presented on a tactile display consisting of a $15 \times 15$ matrix of plastic-tipped point-stimulators, each .05 in. in diameter and spaced .2 in. apart, which poked up from holes in a slightly curved Plexiglas restplate. Subjects rested the underside of the four fingers (excluding the thumb) of the preferred hand on the $3 \times 3$ in. restplate. This display is described further, and a photograph is given, in Kirman (1974c); it was controlled by a Digital Equipment Corporation PDP-11/34 computer. The point-stimulators were activated by miniature solenoids energized by $1.5-\mathrm{msec}$ square-wave pulses of $40 \mathrm{~V}$, which produced clear tactile sensations of "taps" or "vibrations" of moderate 
intensity, depending on the number of pulses delivered in any given stimulus pattern. The repetition rate of pulses was always 100 pulses/sec.

\section{Subjects}

Six undergraduates at Queens College $(3$ males and $3 \mathrm{fe}-$ males) served as subjects, and were paid by the hour for their services. None had been previously involved in experiments on tactile perception.

\section{Stimulus Patterns}

Seven spatiotemporal patterns were investigated: two dots moving horizontally across the array (patterns TD7, TD4, TD3); a horizontal line moving vertically (HL13, HL7, HL5); a swinging line pivoted at the bottom of the array and swinging like a bat (SL13, SL7, SL5); a rotating line that rotated counterclockwise about its midpoint (RL8, RL4, RL2); an expanding square that enlarged from a single point to fill the entire array (ES8, ES4, ES3); an expanding boundary in the shape of a square (EB7, EB4, EB3); and an expanding hole, also in the shape of a square (EH7, EH4, EH3). Each of these unfolding patterns consisted of a series of tactile frames in which the progressive displacement or enlargement of the pattern was produced in discrete steps. The number appended to the abbreviated pattern names (e.g., the "7" in "TD7") indicates the number of steps (or successive frames) used to trace out that pattern over time. Figure 1 illustrates the first two frames (or steps) for five of the patterns (TD7, HL13, SL13, RL8, and ES7) and one intermediate frame for the other two series of patterns (EB and $\mathrm{EH})$. The large dots indicate active stimulators for that frame. For example, TD7 (a) shows the first frame in a two-dot pattern sequence that, as subsequent frames were presented, produced a perception of two dots moving to the left in a straight line. TD7 (b) simply shows the second frame in that pattern sequence.

Each of the above seven pattern shapes was presented with three different numbers of steps. In the case of TD, there was a TD7 pattern (with seven steps), a TD4 pattern (with four steps), and a TD3 pattern (with three steps). Most of the stimulus patterns used seven, four, and three steps, but some involved more steps. Two patterns (HL and SL) were presented with 13, 7, and 5 steps, and one pattern (RL) was presented with 8,4 , and 2 steps. These variations were dictated by the geometry of the tactile array.

\section{Procedure}

All tactile stimulus patterns were presented in a sequence of "frames" much as a motion picture is presented by a series of sequential frames of visual information. Each tactile frame, when
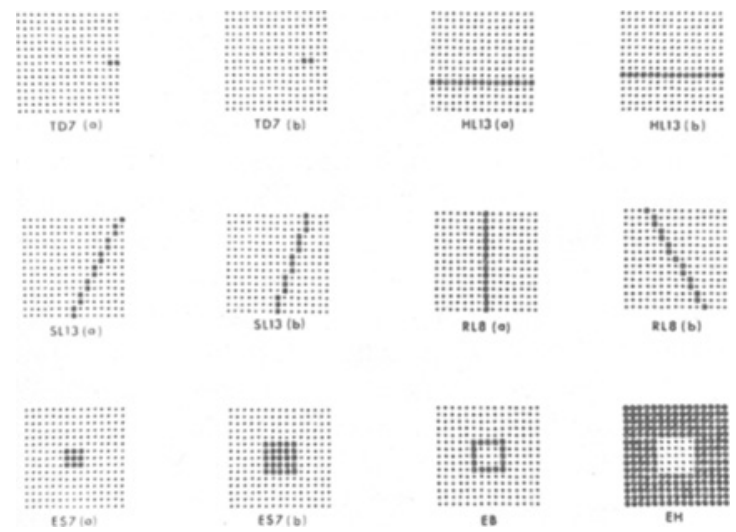

Figure 1. A sample of the array configurations used in each of the stimulus patterns. energized, consisted of the activation of those point-stimulators of the array designated to be "on" for that frame of the stimulus pattern. The stimulus duration of a given spatiotemporal pattern was determined by the number of times each frame in that pattern was repeated (the repetition rate was fixed at $100 / \mathrm{sec})$. The durations used were: approximately $1 \mathrm{msec}(1$ repetition per frame), $10 \mathrm{msec}$ ( 2 repetitions), $20 \mathrm{msec}$ ( 3 repetitions), $50 \mathrm{msec}$ ( 5 repetitions), $100 \mathrm{msec}$ (10 repetitions), and $200 \mathrm{msec}$ ( 20 repetitions). The second temporal variable, interstimulus onset interval (ISOI), the interval between the onset of a given frame in a pattern and the onset of the next frame in that pattern, indicates the rate at which changes in the pattern were stepped into the array. ISOIs of $10,20,30,50,70,100$, 200 , and $400 \mathrm{msec}$ were used. It should be noted that if a given pattern was assigned an ISOI that was shorter than its duration, there resulted a persistence of stimulation from earlier frames that was added to the stimulation from later frames. That is to say, one or more frames actually presented could combine stimulus components from two or more overlapping frame steps of the pattern. Each of the 21 spatiotemporal patterns was presented at all possible combinations (48) of duration and ISOI. It should be emphasized that "duration" in this experiment referred to the length of time a given frame of information (or step of the pattern) was presented, and not to the time required to present the entire spatiotemporal pattern with all of its steps.

Before any data were collected, the subjects were first exposed to one of the more impressively moving patterns (SL13) to acquaint them with the experience of tactile apparent movement and with the apparatus and general procedure. On this occasion, as well as at the beginning of each experimental session, the subject was presented with an ascending series of ISOI values with the 100 -msec duration for the pattern to be investigated during that session. After each such stimulus presentation, the subject was asked to describe, as well as possible, the resulting tactile experience and, in particular, the shape and motion involved. This procedure was continued until each subject correctly described the shape and the motion. When the subject was unable to do that adequately after an entire series had been presented, feedback was given in the form of verbal description by the experimenter, and the subject was allowed to look at a visual display of neon lights which exhibited the same motions that were being tactually displayed. The subject was encouraged to compare the tactile and visual experience, and then, without further visual feedback, was reexposed to the entire tactile series of ISOI values. If the subject now reported acceptable perception of the shape in motion, and had a clear image of what to look for, data collection was begun.

The 21 patterns were presented in a counterbalanced fashion, with only one pattern being presented in any one experimental session (which lasted approximately $1 \mathrm{~h}$ ). For counterbalancing purposes, the patterns were considered as comprising three groups which differed in the nature of motion involved: linear (TD and HL patterns), rotating ( $S W$ and $R L$ patterns), and expanding (ES, EB, and EH patterns). The order of presentation of these three motion-defined groups was counterbalanced across the six subjects, as was also the order of presentation of the two or three patterns within each motion group. Within each pattern shape, the three differing numbers of steps (for example, TD7, TD4, and TD3) were also counterbalanced across subjects.

Within any given experimental session (i.e., for any given pattern), the order of the six durations was randomized. The subjects were presented with all of the ISOls for a given duration in both ascending and descending series, and after each stimulus presentation they were asked whether or not they were able to perceive the given shape in clear smooth motion. For each ascending and descending series, some of the stimuli were repeated so that subjects would not be biased in their movementthreshold judgments by the serial position of a given stimulus in the series. From one to five repetitions, determined and assigned randomly to the eight stimuli in each series, were employed. 
Three ascending and three descending series were used to obtain six estimates of the upper and lower limits of clear and smooth motion. With long ISOIs, movement perception broke down into separate perceptions of successive stimuli. At the lower limit with short ISOIs, either the stimuli appeared to be simultaneous (i.e., not moving) or the movement was too fast for the subject to perceive the shape that was in motion.

After six estimates of upper and lower movement thresholds were determined by this modified method of limits, each subject was again given ascending and descending series, but now extreme stimuli which had never been judged by that subject to produce clear motion were eliminated. Again, up to five random repetitions of randomly selected stimuli were employed in each of these series. After one such up-and-down series was presented with no judgments required, the subject was exposed again to this now more limited range of stimuli (all of which had previously yielded an acceptable experience of motion of that shape for that subject), still using a modified method of limits. On this second exposure, the subject was asked to judge whether each given stimulus was better or worse in the clarity and smoothness of the perception of that shape in motion than the immediately preceding stimulus in the series. By this means, the subject estimated the optimal or best ISOI value for the perception of that moving shape at each duration. Two ascending and and two descending series were used to obtain four such estimates of optimal ISOI values.

\section{RESULTS AND DISCUSSION}

Figures 2 and 3 give a summary in graphic form of all the data, pooled over subjects. The three expanding square shapes (ES, EB, and EH) are presented in Fig-
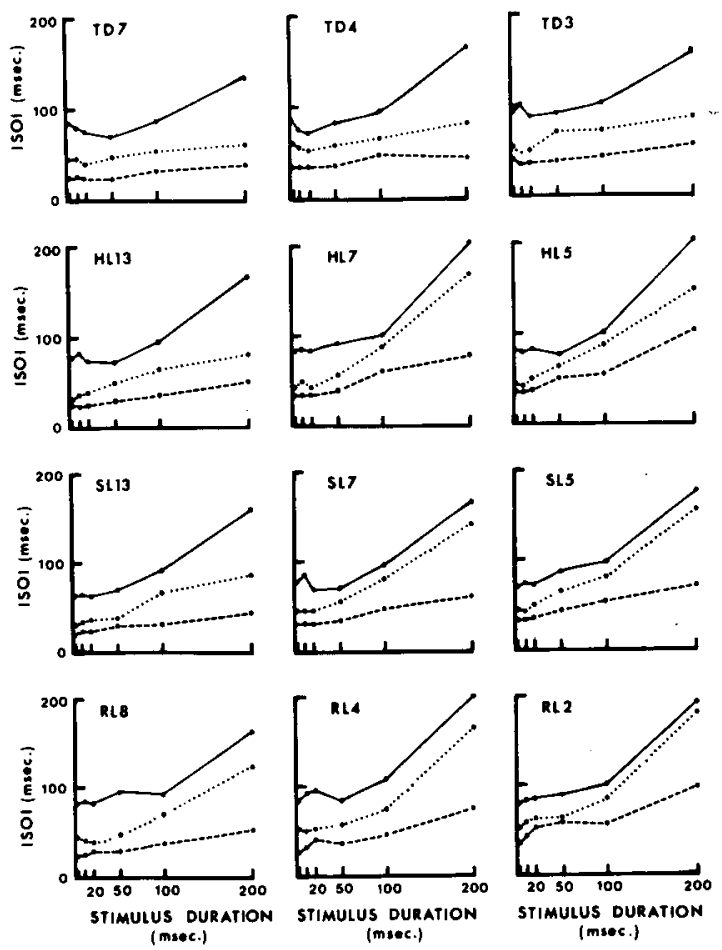

Figure 2. The maximum (solid line), optimum (dotted line), and minimam (dashed line) ISOI values required for good apparent movement plotted as a function of stimulus duration for 12 of the stimulus patterns.
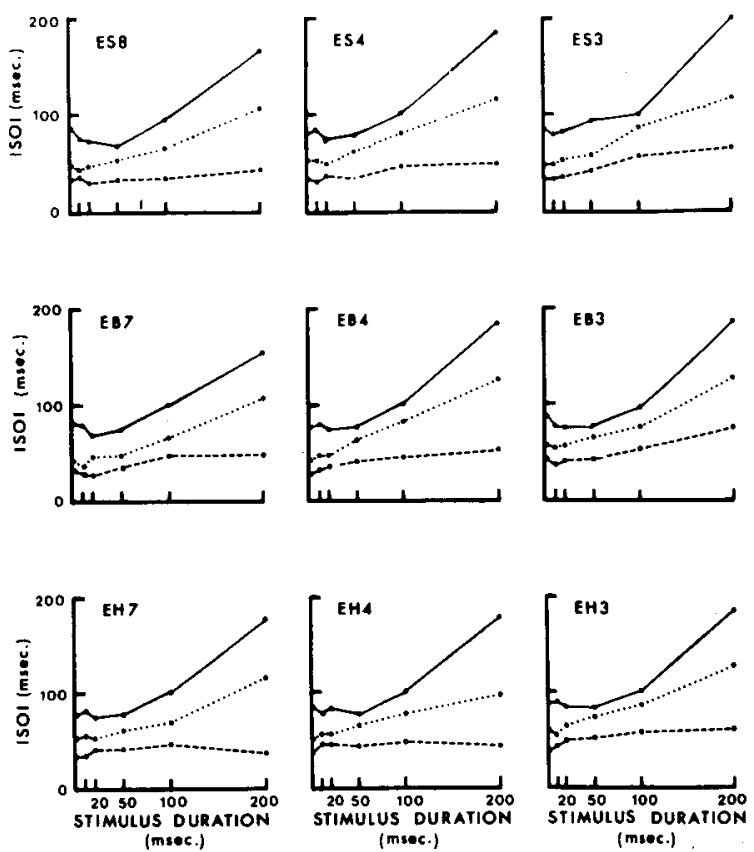

(msec.)

(msec.)

Figure 3. The maximum (solid line), optimum (dotted line), and minimum (dashed line) ISOI values required for good apparent movement plotted as a function of stimulus duration for nine of the stimulus patterns.

ure 3, and the remaining four shapes given in Figure 2. For all seven shapes, the three sets of curves are arranged from left to right in order of decreasing number of steps per pattern. The graphs depict the manner in which maximal, optimal, and minimal ISOI values vary as a function of increasing stimulus duration, for each shape, type of motion, and number of intervening steps. The data here plotted are means of the median judgments for each subject for each condition.

Several analyses of variance (ANOV) using subjects $X$ treatments as error terms were performed to evaluate the role of pattern shape, number of steps, and stimulus duration on the minimum, optimum, maximum, and range (maximum minus minimum) of ISOI values. The results of the most comprehensive ANOV, which included all of the data, are given in Table 1 . A highly significant main effect of duration was found on all dependent variables, while number of steps had very significant main effects on all variables except the range. Pattern shape failed to reveal a main effect on any dependent variable, but the interaction between shape and duration was highly significant for both the optimum and the minimum. A similar pattern was found for the interaction between number of steps and duration. None of the remaining effects were found to be significant, with the exception of the three-way interaction between pattern shape, duration, and number of steps on the optimum.

The significant effects of stimulus duration on the maximum, optimum, and minimum ISOI can be seen in 
Table 1

Results of Analysis of Variance of ISOI Measures Which Included All Stimulus Conditions

\begin{tabular}{|c|c|c|c|c|c|}
\hline \multirow[b]{2}{*}{ Source } & \multirow[b]{2}{*}{$\mathrm{df}$} & \multicolumn{4}{|c|}{ F Value } \\
\hline & & Optimum & Maximum & Range & Minimum \\
\hline Pattern (A) & 6,30 & 1.39 & .71 & .60 & 1.40 \\
\hline Steps (B) & 2,10 & $31.62 \dagger$ & $5.88^{*}$ & 1.13 & $20.08 \dagger$ \\
\hline Duration (C) & 5,25 & $23.64 \dagger$ & $40.30 \dagger$ & $36.66 \dagger$ & $27.25 \dagger$ \\
\hline $\mathrm{AxB}$ & 12,60 & 1.61 & .57 & 1.20 & 1.38 \\
\hline $\mathrm{AxC}$ & 30,150 & $5.11 \dagger$ & 1.35 & 1.01 & $4.27 \dagger$ \\
\hline $\mathrm{BxC}$ & 10,50 & $3.89 \dagger$ & 1.29 & .66 & $4.49 \dagger$ \\
\hline $\mathrm{AxB} \times \mathrm{C}$ & 60,300 & $1.39^{*}$ & .81 & .80 & .87 \\
\hline
\end{tabular}

${ }^{*} p<.025 . \quad t_{p}<.005$.

all of the graphs shown in Figures 2 and 3. After some minor variations at short durations, increasing duration led to increasing ISOI values for all stimulus patterns. These duration effects, pooled over subjects, pattern shape, and number of steps are plotted more clearly for maxima, optima, and minima in Figure 4 and for the range in Figure 5. These observations with respect to stimulus duration are in agreement with earlier findings, both in the tactile sense (Kirman, 1975; Sherrick \& Rogers, 1966) and in vision (Kolers, 1964; Orlansky, 1940). The magnitude of the range of ISOIs over which apparent movement can be obtained has been considered an estimate of the strength of the illusion for given conditions of other variables (Orlansky, 1940). In this context, it is of interest that in 17 different ANOVs which examined various groupings of the data, the range was always very highly significantly influenced by stimulus duration $(\mathrm{p}<.005)$, and never by any other variable or interaction of variables.

Figure 6 gives the effects of number of steps, pooled over subjects, pattern shape, and duration on the maximum, optimum, and minimum ISOI. As we have seen, the decrease in ISOI with increases in number of steps was significant for all three dependent variables. This result is in agreement with previous observations on less complex patterns of tactile movement (Kirman, 1975).

Figure 7 shows the optimum functions for all of the pattern shapes (but one) with approximately the same number of steps (7 or 8) ES7 is omitted only because it so precisely overlaps EH7, which is plotted. This graph does indicate some differences in optima due to pattern shape, especially at longer values of stimulus duration, although omitting TD7 would seem to result in a much more homogeneous group of functions. The results of an ANOV done on only these seven patterns, which had 7 or 8 steps (i.e., that were matched for number of steps), are given in Table 2. Pattern shape by itself, as well as in interaction with duration, did have significant effects here on the optimum and the minimum, but not on the maximum. (As mentioned above, the range was not significantly influenced by any variable other than stimulus duration, and it will therefore be omitted from further descriptions of statistical findings.) Table 3 gives the results of an ANOV identical to the one above except that

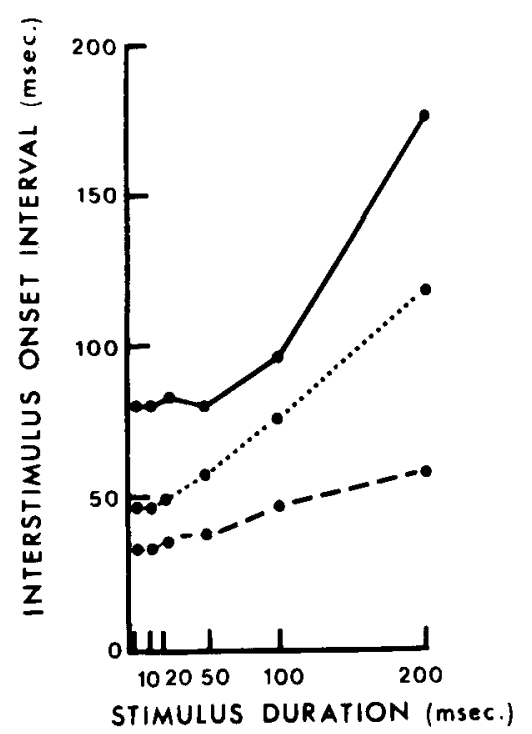

Figure 4. The maximum (solid line), optimum (dotted line), and minimum (dashed line) ISOI values required for good apparent movement plotted as a function of stimulus duration, pooled over all stimulus patterns and numbers of steps.

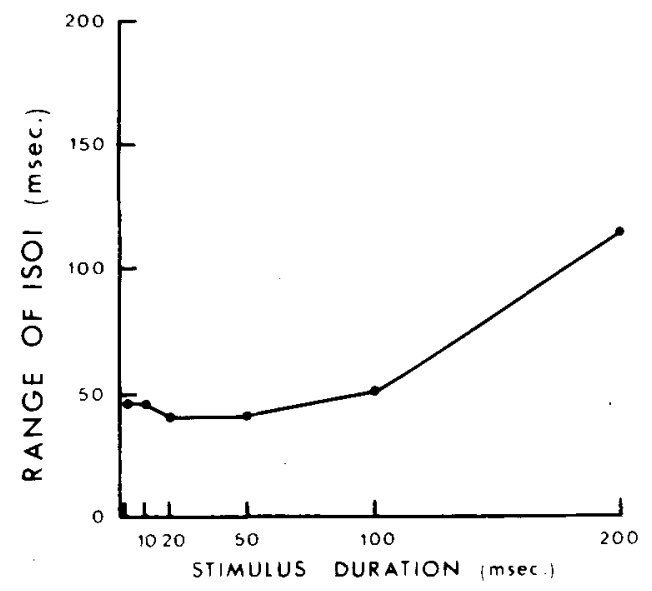

Figure 5. The range of ISOI values required for good apparent movement plotted as a function of stimulus duration, pooled over all stimulus patterns and numbers of steps. 


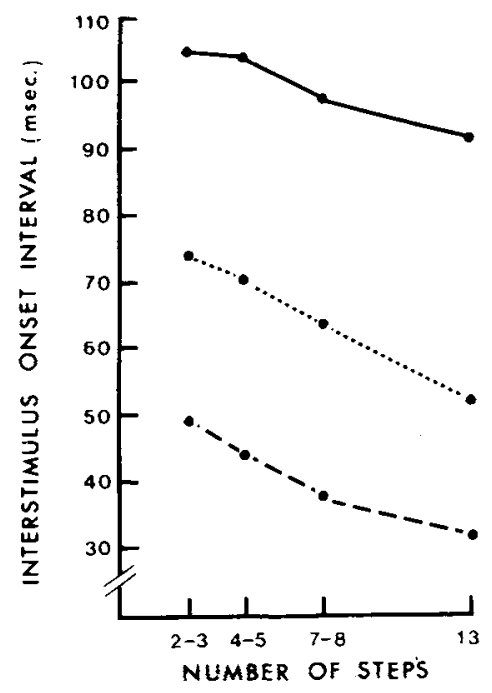

Figure 6. The maximum (solid line), optimum (dotted line), and minimum (dashed line) ISOI values required for good apparent movement plotted as a function of number of steps, pooled over all stimulus patterns and durations.

pattern TD7 was omitted. Here pattern shape failed to produce any significant main effects and its interaction with duration resulted in a significant effect only for the minimum. It would appear that the discrepancy between TD7 and the other pattern shapes accounted for most of the observed effects due to pattern variations, though some differences do remain due to the other pattern differences.

A series of ANOVs was done to check on specific comparisons between selected patterns. The following comparisons were made: TD7 with HL7, SL7 with RL8, TD7 with RL8, HL7 with SL7, TD7 with SL7, HL7 with RL8, and the three expanding patterns EB7, ES7, and EH7 with each other. Of all these comparisons, a main effect due to pattern shape was found only for TD7 vs. HL7 (maximum, $p<.01$; optimum, $p<.01 ;$ minimum, $p<.005$ ), and for the minimum only in the HL7 vs. SL7 comparison $(p<.05)$. The data for the two patterns that differed the most, TD7 and HL7, are replotted in Figure 8 . The two sets of curves differ from each other not only in average height, but also in their slopes. The ANOV comparing the two patterns found a highly significant interaction between pattern shape and duration for the maximum $(p<.005)$, optimum $(p<.005)$, and minimum $(\mathrm{p}<.005)$.

In the remaining comparisons between pattern shapes, a few scattered significant interactions between pattem and duration were found: TD7 vs. RL8 optima ( $p<.005)$; HL7 vs. SL7 minima $(p<.05)$; TD7 vs. SL7 maxima $(\mathrm{p}<.025)$, optima $(\mathrm{p}<.005)$, and minima $(\mathrm{p}<.01)$; HL7 vs. RL8 optima $(\mathrm{p}<.025)$ and minima ( $<<.05)$; and EB7 vs. ES7 vs. EH7 minima ( $<<.05)$. All other pattern $X$ duration interactions were insignificant. It is clear that the two patterns TD7 and HL7, in their mutual differences and in their differences from other patterns, accounted for almost all of the effects due to pattern shape.

It is worthwhile to look more closely at the pattern differences between TD7 and HL7. Both patterns consisted of seven steps, and the interstimulus distance between successive steps was .4 in. in both cases. The difference lay in the fact that pattern TD7 was a series of two-dot stimuli whose successive presentation was equivalent to a straight line's being drawn across the skin surface, while HL7 (made up of a series of horizontal 15-point lines moving in a vertical directionfirst up and then down) could be compared to a straightedge's being scraped up and down the skin surface in a direction perpendicular to its orientation. It is reasonable to interpret the observed discrepancies between the two as primarily due to differences in the ease with which the two shapes could be perceived, rather than as a result of differences in the motion component of subjects' judgments. It was noted in the description of the procedure above that subjects were instructed to report positively only when they could perceive that particular shape clearly in motion. Considering pattern HL7, it is evident that, as duration is increased (i.e., previous steps of the pattern persist along with later steps), the entire pattem approaches a solid rectangular block of active stimulators in which the perception of a thin horizontal line may be obscured at short ISOIs. With pattern TD7, however, subjects may have reported clear object perception when they felt a clean horizontal line rather than separable dot pairs in motion. There is no ISOI that is too rapid for the clear perception of this horizontal line-which is readily perceivable even with an ISOI of 0 msec. It is likely, therefore, that the curves for pattern TD7 reflect the limits for the perception of

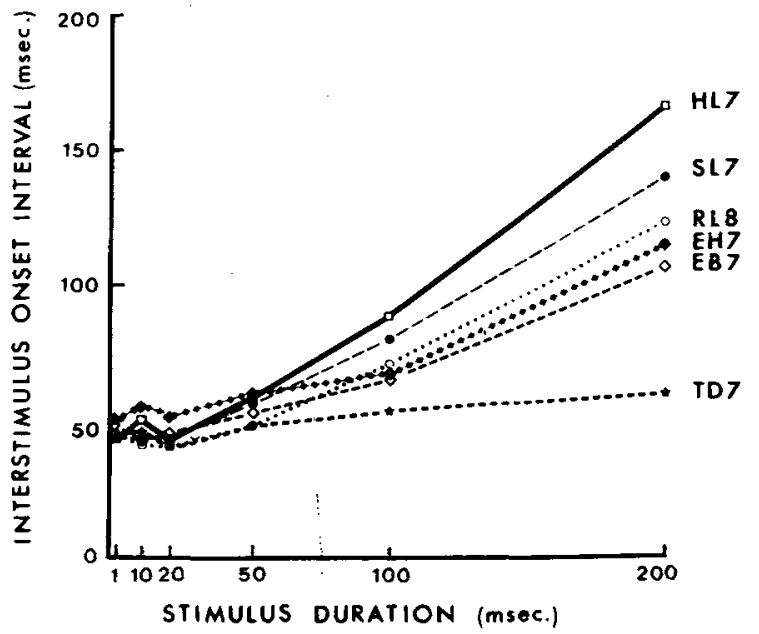

Figure 7. The optimum ISOI values required for good apparent movement plotted as a function of stimulus duration for each of the stimulus patterns (except EB7-see text) with seven or elght steps. 
Table 2

Results of Analysis of Variance of ISOI Measures Which Included Only Patterns Having 7 or 8 Steps

\begin{tabular}{lccccc}
\hline & & \multicolumn{4}{c}{ F Value } \\
\cline { 4 - 6 } Source & df & Optimum & Maximum & Range & Minimum \\
\hline Pattern (A) & 6,30 & $2.73^{*}$ & 1.41 & .71 & $2.94^{*}$ \\
Duration (B) & 5,25 & $18.86 \dagger$ & $33.21 \dagger$ & $17.93 \dagger$ & $23.82 \dagger$ \\
AxB & 30,150 & $2.85 \dagger$ & 1.53 & 1.40 & $4.44 \dagger$ \\
\hline
\end{tabular}

$* p<.025 . \quad t p<.005$

Table 3

Results of Analysis of Variance of ISOI Measures Which Included Only Patterns Having 7 or 8 Steps With TD7 Omitted

\begin{tabular}{lccccc} 
& & \multicolumn{3}{c}{ F Value } \\
\cline { 3 - 5 } Source & df & Optimum & Maximum & Range & Minimum \\
\hline Pattern (A) & 5,25 & 1.61 & .98 & .77 & 1.89 \\
Duration (B) & 5,25 & $18.88 \dagger$ & $32.51 \dagger$ & $18.54 \dagger$ & $23.90 \dagger$ \\
AxB & 25,125 & 1.54 & 1.17 & $4.65 \dagger$ & 1.35 \\
\hline
\end{tabular}

$t p<.005$.

something like "pure motion" unconstrained by the limits of object recognition.

Despite these few differences due to pattern shape and those earlier attributed to number of steps, looking again over the large number of graphs in Figures 2 and 3 for stimuli that vary from two dots to swinging lines to expanding holes and that vary in number of successive stimuli from 2 to 13 , what is most striking about the graphs is their similarity. They all describe very similar functions of ISOI. Furthermore, they are sufficiently similar in terms of absolute ISOI values for each duration for it to be possible to draw an ISOI-duration function that would be included within the range of acceptable movement for all 21 patterns. The relative lack of influence of shape and shape perception on these apparent movement functions is underscored by the very similar functions obtained for the expanding hole (a shape that was very difficult for our subjects to detect or describe at all), the expanding square (which was much more easily detectable), and the rotating line (which could be very clearly perceived).

The practical consequence of these similarities is that a tactile code may be designed with confidence that stimulus integration resulting from apparent movement will be maintained over variations in tactile patterns-as long as the proper temporal intervals between stimuli and the proper durations have been arranged. This not only simplifies the design of any arbitrary tactile code that seeks to utilize apparent movement, but insures that the output of any speech-to-tactile transformation could maintain perceptual integration through apparent movement regardless of changes in the nature of the tactile patterns produced.

It is also of some theoretical interest that shape variations have so little influence on the temporal conditions for tactile apparent movement. Kolers has observed that in the visual modality also "while disparity of shapes is not altogether without effect, its influence upon the ease
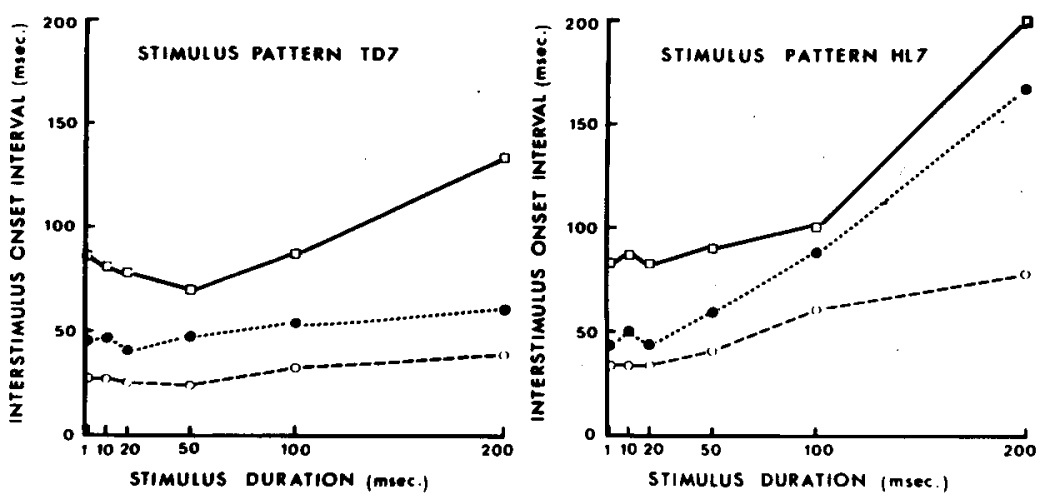

Figure 8. The maximum (solld line), optimum (dotted line), and minimum (dashed line) ISOI values required for good apparent movement plotted as a function of stimulus duration for the two patterns, TD7 and HL7. 
of seeing motion is quite small" (Kolers, 1972, p. 50). Kolers related these observations, originally made by him and Pomerantz (1971) and supported as well by Anstis (1970), to a distinction made long ago by Wertheimer between the perception of "pure motion" (phi) and object perception. In Wertheimer's words, "The phi phenomenon [is] separable from the [processes mediating the] appearance of the two stimulus objects" (quoted in Kolers, 1972, p. 11). This distinction is in accord with the inference that the mechanisms underlying the perception of movement are independent and separable from those underlying the perception of shape. Anstis (1970) and Kolers (1972) suggest this for vision, and the observations made here support such a notion for the tactual modality as well. Indeed, several studies have shown that the tactile sense and the visual sense obey very similar laws in the perception of apparent motion (Kirman, 1974a; Sherrick \& Rogers, 1966), while it is obvious that the two senses are highly dissimilar with respect to shape perception. This similarity in movement perception and disparity in shape perception is a further argument for distinct mechanisms underlying them. It is also reasonable to infer that the relative superiority of movement perception over shape perception in the skin suggests that the skin is a closer evolutionary descendant of primordial sensory capacity than is the eye, and that the mechanisms for movement perception are more primitive than those for the perception of shape.

\section{REFERENCES}

Anstis, S. M. Phi motion as a subtraction process. Vision Research, 1970, 10, 1411-1430.

Anstis, S. M. Apparent movement. In R. Held, H. Leibowitz, and H.-L. Teuber (Eds.), Handbook of sensory physiology (Vol. 8): Perception. New York: Springer, 1978.

KInman, J. H. Tactile communication of speech: A review and an analysis. Psychological Bulletin, 1973, 80, 54-74.

Krnman, J. H. Tactile apparent movement: The effects of interstimulus onset interval and stimulus duration. Perception \& Psychophysics, 1974, 15, 1-6. (a)

KIRMAN, J. H. Tactile apparent movement: The effects of number of stimulators. Journal of Experimental Psychology, 1974, 103, 1175-1180. (b)

Kirman, J. H. Tactile perception of computer-derived formant patterns from voiced speech. Journal of the Acoustical Society of America, 1974, 55, 163-169. (c)

Kirman, J. H. The effect of number of stimulators on the optimal interstimulus onset interval in tactile apparent movement. Perception \& Psychophysics, 1975, 17, 263-267.

Kole rs, P. A. The illusion of movement. Scientific American, $1964,211,98-106$.

Kole rs, P. A. Aspects of motion perception. Oxford: Pergamon Press, 1972.

Kolers, P. A., \& Pomerantz, J. R. Figural change in apparent motion. Journal of Experimental Psychology, 1971, 87, 99-108.

Orlansky, $J$. The effect of similarity and difference in form on apparent visual movement. Archives of Psychology, 1940, 35, No. 246.

Sherrick, C. E., \& Rogers, R. Apparent haptic movement. Perception \& Psychophysics, 1966, 1, 175-180.

(Manuscript received March 8, 1982; revision accepted for publication March 8, 1983.) 